\title{
La teoría decolonial: buscando la identidad en el mercado académico
}

The Decolonial Theory: Searching for the Identity in the Academic Market

A teoria descolonial: procurando a identidade no mercado acadêmico

\section{Jeff Browitt}

UNIVERSITY OF TECHNOLOGY, SYDNEY, AUSTRALIA

Profesor del programa de Estudios Internacionales en la University of

Technology, Sydney, Australia. PhD Monash University, Australia.

Autor de Contemporary Cultural Theory (Routledge, 2002, con

A. Milner), Rubén Darío: cosmopolita arraigado (IHNC, 2010, con W. Mackenbach), Practising Theory: Pierre Bourdieu and the Field of Cultural Production (UP Delaware, 2004, con B. Nelson); y dos traducciones: A New Catechism for Recalcitrant Indians [Nuevo catecismo para indios remisos] de Carlos Monsiváis (FCE, 2010); y Disciplinando a los salvajes, violentando las disciplinas [Disciplining the Savages, Savaging the Disciplines] de Martin Nakata (Aboriginal Studies Press \& Abya Yala Editores, 2014, con N. Castrillón). Correo electrónico: Jeffrey.browitt@uts.edu.au

\footnotetext{
Artículo de reflexión
}

Este artículo se basa en una ponencia presentada en el congreso de Ailasa (Association of Iberian and Latin American Studies of Australasia) en julio de 2010: "Shopping for Identity, or Can the Intellectual Be Subaltern Too?".

Documento accesible en línea desde la siguiente dirección: http://revistas.javeriana.edu.co

doi:10.11144/Javeriana.CL18-36.tdbi 


\section{Resumen}

Los proponentes del discurso decolonial latinoamericano entran en contradicción performativa cuando utilizan las herramientas de la teoría crítica europea para deconstruir el discurso de la modernidad eurocéntrica: tratan de poner en cuarentena sus propias construcciones discursivas e ideológicas y protegerlas de la misma revisión. Ciegos a las aporías de la teoría, piensan que pueden tomar una posición epistemológica moralmente superior y trascendente a través de su contacto con los mundos indígenas y afrodescendientes. Este proceso de apropiación ideológica simplemente invierte los opuestos simplistas que los teóricos decoloniales dicen que quieren evitar.

Palabras clave: América Latina; decolonial; epistemología; indígena; modernidad

\section{Abstract}

The proponents of Latin American decolonial discourse enter into performative contradiction when they utilize the tools of European critical theory to deconstruct the discourse of Eurocentric modernity, while trying to quarantine their own discursive and ideological constructions from review by that selfsame European critical theory. Blind to decolonial theory's aporias, they think they can take a morally superior and transcendental epistemological position through their contact with indigenous and Afrodescendent worlds. This process of ideological appropriation merely inverts the simplistic binary opposites that the decolonial theorists claim they wish to avoid.

Keywords: decolonial; epistemology; indigenous people; Latin America; modernity

\section{Resumo}

Os proponentes do discurso descolonial latino-americano entram em contradição performativa quando utilizam as ferramentas da teoria crítica europeia para desconstruir o discurso da modernidade eurocêntrica: tentam pôr em quarentena suas próprias construções discursivas e ideológicas e protege-las da própria revisão. Cegos às aporias da teoria, acham que podem adotar uma posição epistemológica moralmente superior e transcendente através do seu contato com os mundos indígenas e afrodescendentes. Este processo de apropriação ideológica simplesmente inverte os contrários simplistas que os teóricos descoloniais dizem querer evitar.

Palavras-chave: América Latina; descolonial; epistemologia; indígena; modernidade

\section{Cómo citar este artículo:}

Browitt, Jeff. "La teoría decolonial: buscando la identidad en el mercado académico". Cuadernos de Literatura 18.36 (2014): 2546. http://dx.doi.org/10.11144/Javeriana.CL18-36.tdbi 
Para los teóricos de la decolonialidad, hay una serie de términos/conceptos claves que funcionan como blancos de su crítica: Europa, eurocentrismo, Occidente, Modernidad, Ilustración, capitalismo, racionalismo, universalismo, Estado, blanco, etc. A dichos términos oponen sus propios neologismos: "colonialidad del poder", "hermenéutica pluritópica", "border gnosis", "diferencia epistémica colonial", "pluriversidad" y "diversalidad", "plurinacionalidad" y "buen vivir" (sumak kawsay), entre otros. Walter Mignolo, Enrique Dussel, Rodolfo Kusch, Aníbal Quijano, Nelson Maldonado-Torres, Ramón Grosfoguel, Catherine Walsh, Santiago Castro-Gómez y otros quieren llamar la atención hacia el hecho de que la lógica colonial, que fue de la mano con la expansión y el desarrollo capitalista industrial europeo, todavía está vigente como un proceso material real y como una ideología inconsciente y sedimentada en la base de los imaginarios políticos y culturales, incluso en el mundo no-europeo. Aun el discurso postcolonial, aunque oposicional en su génesis, se considera a fin de cuentas atado al pensamiento colonial ${ }^{1}$. La postura de los teóricos de la decolonialidad también impacta en la legitimidad del latinoamericanismo académico norteamericano, la geopolítica del conocimiento y la política del locus de enunciación.

Los decolonialistas están ansiosos de proponer una "diferencia colonial" que evite dualismos culturales e ideológicos. Sin embargo, no siempre obedecen sus propios dictados ya que una y otra vez ponen en práctica un tipo de interpretación estática y reductora de Europa, Occidente, Modernidad, basada en binarios fijos y demasiado fuertes, como centro-periferia, Europa-no Europa, modernidad-el otro de la modernidad y modernidad-tradición, que

1 La objeción a los estudios poscoloniales está basada en una caricatura de los mismos que utiliza argumentos endebles fundados en binarios estáticos: "Postcoloniality shares with the world-system approach a critique of developmentalism, of Eurocentric forms of knowledge, of gender inequalities, of racial hierarchies, and of the cultural/ideological processes that foster the subordination of the periphery in the capitalist world-system. However, the critical insights of both approaches emphasize different determinants. While postcolonial critiques emphasize colonial culture, the world-system approach emphasizes the endless accumulation of capital at a world-scale. While postcolonial critiques emphasize agency, the world-system approach emphasizes structures. Some scholars of the postcolonial theory such as Gayatri Spivak (1988) acknowledge the importance of the international division of labour as constitutive of the capitalist system while some scholars of the world-system approach such as Immanuel Wallerstein acknowledge the importance of cultural processes such as racism and sexism as inherent to historical capitalism. However, the two camps in general are still divided over the culture vs. economy and the agency vs. structure binary oppositions. This is partly inherited from the 'two cultures' of western knowledge that divide the sciences from the humanities, premised upon the Cartesian dualism of mind over matter" (Grosfoguel, "Transmodernity" 10). Véanse también Castro-Gómez y Grosfoguel; Grosfoguel y Saldívar. 
no corresponden a la realidad contemporánea - las fronteras entre ellos han sido borrosas por mucho tiempo-. En nuestra época las relaciones de poder y dominación son mucho más fluidas y movedizas y, al igual que el concepto de "modernidad", no pueden reducirse solamente a la "colonialidad" (aunque la colonialidad o el colonialismo sean efectos duraderos de dicha modernidad europea). No obstante, para los decolonialistas la modernidad/colonialidad es un artículo de fe inquebrantable, razón por la cual culpan a todo lo europeo y todo lo "moderno" por los males del mundo. Limitan el desarrollo de la modernidad casi exclusivamente al pillaje de las Américas (que sin duda es un elemento clave), pero sin reconocer ninguna de las dinámicas que estaban dentro del desarrollo de la modernidad europea mucho antes del punto inicial simplista y reduccionista de la colonización ibérica. Mignolo, por ejemplo, propone una lectura de la modernidad europea (o por lo menos su discurso autojustificante) como una fuerza y una epistemología que han reprimido las formas de conocimiento y de ser de los indígenas. Está bien. Es indiscutible. No obstante, esas otras formas de conocimiento no son prístinas y no están congeladas; eso sería una representación a fin de cuentas ingenua y condescendiente de las culturas indígenas (que linda con el racismo que los decolonialistas quieren evitar), porque hablan como si los indígenas no hubieran evolucionado o como si de alguna manera sus culturas fueran necesariamente igualitarias, no competitivas ("complementarias") y justas, simplemente por ser no-europeas.

El uso del concepto y del discurso de la modernidad, positivamente o en oposición a ello, está cargado de problemas por definición. Solo puede sostenerse si se adhiere a los criterios utilizados para nombrarlo. Pero lo que representa la modernidad puede ser renombrado o reagrupado bajo otros conceptos y otras lecturas de la historia con otros fines políticos, no solo con los fines de dominación. Entonces la validez de la lectura de la modernidad occidental de los teóricos de la decolonialidad únicamente puede mantenerse en la medida en que la definición se conserva en pie. Pero si es expuesta como parcial, unilateral, altamente selectiva y basada en el mismo pensamiento binario que dichos teóricos censuran en la tradición intelectual europea, entonces su narrativa histórico-política entra en dificultades.

Si aceptamos que el concepto de modernidad es útil para los debates filosóficos y políticos, entonces tiene muchas genealogías, muchas lecturas. La modernidad europea no puede ser reducida solo al colonialismo: había transformaciones profundas en marcha en las ciudades y en las religiones europeas antes del periodo colonial en América Latina: el nominalismo en la religión cristiana y el individualismo en la política surgen de los asuntos de la cristiandad 
y su idea de la salvación individual y de la polis griega. En Passage to Modernity, por ejemplo, Louis Dupré identifica un "modo" o "ánimo" de la modernidad, y aunque un sentimiento de modernidad iba a la par con la expansión europea y contribuía a su autovalidación entre las élites europeas políticas, económicas y culturales (por lo menos después del siglo XVIII), no puede explicar esas otras transformaciones anteriores al colonialismo europeo dentro del marco de lo que comúnmente entendemos por tal. Dicho sea de paso, los ingleses practicaron el colonialismo en Irlanda en el siglo XIII, así que el colonialismo no es simplemente un proceso post-1492; ni, por definición, es solamente un fenómeno europeo. Si aceptamos que el colonialismo es la extensión del control, típicamente por medio de la violencia, sobre otro territorio y otro grupo humano y el establecimiento de relaciones de tributo, entonces el colonialismo ha sido universal por milenios. ¿Qué fueron los imperios incaico y azteca si no imposiciones violentas sobre otros pueblos?

"Modernidad" es considerado sinónimo de términos como "Europa" y "Occidente", zonas geográficas en las cuales se desarrolló la modernización socioeconómica. Pero los países de la llamada "América Latina", por vago que sea dicho término, son también occidentales a su manera, aunque, como es bien sabido, hay muchos grupos indígenas o de descendencia africana en varias etapas de occidentalización y algunos poco occidentalizados. Pero lo que no se puede hacer es, conveniente y tajantemente, separar Europa de América Latina y poner a andar el reloj de la historia al revés y restaurar algún mundo anterior a la colonización. En el mismo sentido, la modernidad no puede reducirse a un mote simplista. Es verdad que la modernidad europea tiene en su base sistemas de dominación como el capitalismo, la burocratización de la vida, el racismo y el colonialismo; pero tiene unos elementos emancipatorios que también salieron de la misma Ilustración y que van en contra de las tendencias negativas: la igualdad y la solidaridad, discursos sobre los "derechos" a la libertad por oposición a la tiranía y las jerarquías feudales y eclesiásticas, derechos que los indígenas mismos movilizan para formular sus reclamos. Mauricio Domingues afirma:

hay que ver la modernidad como un fenómeno de doble filo: la dominación pero también la emancipación. Contra su cosificación, sugiero que la veamos como tejido de múltiples y contingentes pasos modernizantes [...] Los puntos de vista de Mignolo tienen dos equivocaciones básicas: 1) una visión reductora de la modernidad en la cual solo la dominación aparece como relevante, y no se permite la ambivalencia; y 2) la idea de que solo lo que no es moderno -o por lo menos tiene una relación ambigua con la modernidad - tiene valor en América Latina. (114, traducción mía) 
Y es más: el chovinismo etnocéntrico, el patriarcado, el racismo y el sentimiento de superioridad cultural no son exclusivos de pueblos europeos ni de la modernidad. Maldonado-Torres, por ejemplo, ve la utilidad de aspectos de la modernidad para la teoría decolonial: "las apropiaciones críticas y creativas de ciertas ideas modernas, junto con otros múltiples marcos conceptuales que pueden forjar un futuro menos opresivo [...] quitando de la modernidad sus elementos y parcialidades colonizadores" (5). Bien, pero desgraciadamente casi nunca oímos de los aportes positivos de la modernidad o la Ilustración en el discurso decolonial, solo lo negativo.

Otro ángulo desde el cual Mignolo ataca la modernidad europea, y utiliza una cosmogonía indígena andina para tal propósito, es el del concepto de "cosmopolitismo". En "Cosmopolitanism and the De-Colonial Option" (2010), comienza con una genealogía a partir de Kant y considera que sus opiniones sobre el cosmopolitismo son eurocéntricas (este tipo de crítica del cosmopolitismo de Kant era un lugar común antes de Mignolo, por supuesto). Mignolo está de acuerdo en que el núcleo de la idea del cosmopolitismo es bueno - ciudadanos iguales en todo el mundo-, sin embargo aboga por una variante de-colonial y no eurocéntrica. Cita Cosmopolis. The Hidden Agenda of Modernity (1990), de Stephen Toulmin, para respaldar su propuesta. Según Mignolo, la argumentación de Toulmin es la siguiente: en los siglos XVI y XVII, el Cosmo-polis fue creado siguiendo las líneas de representación del cosmos que la física y la astronomía estaban construyendo en ese periodo. La sociedad europea estaba siendo reconstruida tras la guerra de los Treinta Años (1618-1648), la guerra que llevó al Tratado de Westfalia y el reconocimiento del principio de la soberanía del Estado, que fue un precursor del sistema del Estado-nación moderno y, posteriormente, de la meditación de Kant sobre el cosmopolitismo. Mignolo resume el pensamiento de Toulmin así:

[el nuevo Cosmopolis europeo] se basó en dos pilares o principios: la estabilidad y la jerarquía. La estabilidad se aplicó a las interrelaciones entre las naciones soberanas; la jerarquía a la organización interna de la sociedad [...] La organización jerárquica de cada nación-(polis)-Estado seguirá el modelo proporcionado por la ley del cosmos; las relaciones de estabilidad entre las naciones-Estado también se modelarán en la ley de la naturaleza (cosmos) que sirve de modelo para la organización de cada Estado dentro de sí mismo (polis). (Mignolo, “Cosmopolitanism” 115, traducción mía)

Según Mignolo, esta versión del cosmopolitismo y sus fundamentos filosófico-políticos eurocéntricos "deberá responder por sus crímenes: el fundamento mismo del cosmopolitismo, según lo previsto por Kant y explicado por Toulmin, 
estaba en complicidad con la formación de las potencias imperiales europeas y con la expansión europea en América, África y Asia" ("Cosmopolitanism" 116117). Pero lo que es bastante extraño y fantasioso en esta declaración simplista, además de la retórica ("crímenes"), es la idea de que las personas involucradas en la conversación o las prácticas cosmopolitas actualmente se adhieren a esta versión del siglo XVII (y no se quiere decir que no haya algunos); o peor aún, que las charlas sobre el cosmopolitismo hoy en día realmente significan algo para la mayoría de la gente más allá de los intelectuales, lo que no es el caso, aunque el problemático discurso sobre el multiculturalismo linda a veces con los valores del cosmopolitismo (tanto la variante kantiana como la no kantiana). Esto no es un problema para Mignolo, hecho que se ilustra en su reflexión acerca de la reescritura de las Constituciones de Bolivia y Ecuador de la siguiente manera:

y todo el debate sobre el Estado plurinacional no es otra cosa que la continuación del problema que Guamán Poma vio emerger hace 500 años, cuando el territorio de los incas y aimaras se convirtió en una mezcla de etnias. Su tratado político-colonial era, y sigue siendo, ejemplar: no propuso la coexistencia con el enemigo. Por el contrario, la idea misma de enemigos no venía a su mente. Por lo tanto, uno de los primeros pasos del cosmopolitismo decolonial es deshacerse de la idea de amigos y enemigos, en la que la política encuentra su razón de ser. ("Cosmopolitanism" 118)

Ojalá fuera así, pero el tenor del discurso decolonial sugiere otra cosa. Sigue Mignolo: "el problema no es de menor importancia y toda la idea del cosmopolitismo decolonial está en juego" ("Cosmopolitanism" 118). En otras palabras, Mignolo ha inflado el debate aquí: se ve a sí mismo en el centro de la cuestión (obviamente junto con otros filósofos latinoamericanos como Enrique Dussel y Aníbal Quijano) y la única salida válida al futuro viene a ser una apropiación de una versión de una cosmogonía andina que acabará con las enemistades entre los seres humanos. Bien puede ser, y suena atractivo, pero ahora cuando supuestamente tenemos que rechazar los universalismos y dado que, según los decolonialistas, los europeos se apropiaron de los habitantes de América Latina para sus proyectos utópicos, los intelectuales decoloniales radicalizados no pueden atribuirse el derecho de hacer lo mismo, sin entrar en otra contradicción performativa.

$\mathrm{Al}$ igual que muchos estudiosos decolonialistas que trabajan en temas indígenas, Mignolo presenta una versión rosada de la historia precolombina. No menciona la sangrienta expansión imperial de los imperios inca y azteca antes del imperialismo europeo, como si hubiera algún tipo de paraíso indígena precolombino no problemático, lo que refuerza aún más la idea de que el mundo indígena 
era estático. Mignolo habla de la matriz colonial de poder, la "gubernamentalización" de los pueblos de las colonias europeas (para usar el término foucaultiano de gestión, control y disciplina de la población). Está bien, pero también había una matriz colonial de poder sobre los pueblos conquistados por los incas y aztecas antes de la llegada de Colón, aunque esa frase no se usa específicamente en relación con la situación precolombina. Puede ser que los imperios azteca e inca no tuvieran una doctrina explícita de soberanía al estilo europeo, tal como la describe Mignolo, pero tenían un sentimiento de soberanía de facto. ¿Cómo se puede subordinar a la gente y defender el territorio si no? Se necesita una justificación para la dominación. Los imperios precolombinos tenían sus propios rituales y jerarquías sociales para eso; están inscritos en sus monumentos. Y, por supuesto, la expansión de imperios a lo largo de la historia nunca ha sido práctica exclusiva de los europeos. Mignolo no dice nada sobre eso tampoco.

Además de la problemática idea de la modernidad, esa región a la que nos referimos como América Latina viene a ser occidental desde 1492, aunque obviamente a su manera: un occidentalismo diferente al europeo, al de los Estados Unidos y al de Australia. Hay, por supuesto, una gran excepción: los pueblos indígenas, que, contra viento y marea, han logrado conservar en menor o mayor medida parte de sus tradiciones y creencias, sus relaciones sociales y sus patrones de tenencia de tierras. Aun así, hay pocos indígenas que puedan decir que no han sido contaminados por la modernidad europea en alguna medida. En su libro de 1994, Amaryll Chanady rechaza la imagen de los indígenas como "salvajes nobles al estilo de Rousseau, quienes no desean más que el vivir en la simple comunión con la naturaleza, no contaminados por los vicios de la civilización moderna" (xxviii, traducción mía). Cita a Asunción Ontivero Yulquila, en aquel entonces coordinador general del Consejo Indio de Sudamérica:

Bajo ningún punto de vista, la indianidad pretendió ni pretende marginarse de la evolución de la humanidad; menos aún refugiarse en un conservadurismo cultural, cuidando solamente nuestros monumentos históricos o parques nacionales [...] La indianidad jamás estuvo ni está contra las ciencias de Occidente o de Oriente. (Cit. en Chanady xxviii, cursiva en el original)

Aunque haya grupos indígenas que sí quieren quedarse alejados de todo lo moderno, Ontivero Yulquila complica la versión estereotípica que propaga el discurso decolonial. Según él, los indígenas no están necesariamente en contra de la modernidad, la europea o cualquier otro tipo; quieren "una democracia de culturas", "la autodeterminación" y "la liberación integral" (cit. en Chanady xxviii). Pero alinearse con ellos como intelectual mestizo radicalizado y apropiarse 
de sus cosmogonías para fines intelectuales, políticos y profesionales es muy problemático. Además, los distintos grupos indígenas y sus discursos no son un bloque homogéneo y estático, ni tienen un solo punto de vista. Algunos quieren acercarse a la modernidad por su propia voluntad; es decir, quieren "indigenizar" la modernidad, comoquiera que entendamos este término. No se ven a sí mismos como culturas congeladas en el tiempo, o agarrados de una concepción estática de su cultura y de sí mismos. ¿Quién había oído sobre el sumak kawsay (vivir bien), por ejemplo, hasta que ciertos políticos y unos cuantos activistas indígenas e intelectuales formados en discursos occidentales lo encontraron útil para la nueva constitución ecuatoriana en 2008? Hay que hacer una genealogía de este concepto e investigar hasta qué punto era de veras un concepto bien conocido y utilizado por los indígenas andinos antes de su aparición en el discurso de Alianza País.

En un reciente dossier sobre el discurso del "buen vivir" o sumak kawsay, Víctor Bretón, David Cortez y Fernando García subrayan su naturaleza como "guía programática contra la modernidad occidental en crisis [...] interpretado por numerosos analistas críticos como una ruptura conceptual en toda regla para con las interpretaciones tópicas (euronorteamericanocéntricas) del progreso, el crecimiento y sus corolarios" (9-10) que provee "nuevos marcos referenciales con qué normativizar la convivencia en sociedades poscoloniales". Aunque la noción del "buen vivir" se remonta por lo menos a 2000, "fue recogida por primera vez en un documento oficial del Estado ecuatoriano: el Plan Nacional de Desarrollo 2007-2010", y pasó a ser "el eje vertebrador de la nueva Constitución de la República, aprobada en referéndum por la mayoría del pueblo ecuatoriano" (10). En contraste con las afirmaciones autovalidadoras del discurso y sus promotores, Bretón, Cortez y García hacen hincapié en la vaguedad y naturaleza sobreelástica del concepto:

[...] la dificultad para entender a qué se suele apelar cuando se habla de sumak kawsay. Se ha dicho, por ejemplo, que se trata de un concepto emanado de las cosmovisiones ancestrales de los pueblos indígenas (y afrodescendientes en algunas acepciones del vocablo) (Walsh, 2009), de una propuesta para avanzar hacia un tipo de sociedad diferente a partir de categorías como la reciprocidad, la convivencialidad, la armonía con la naturaleza (sostenibilidad) (Huanacuni, 2010), la complementariedad, la desmaterialización de la vida y, en suma, la ruptura para con la visión unilineal del progreso y el desarrollo inmanente a la visión occidental hegemónica (Gudynas, 2009 y 2011; Unceta, 2009). Un proyecto y un camino que habrá de concretarse en la verdadera construcción de un Estado plurinacional en el que el diálogo de saberes sustituya el predominio de la racionalidad científica que ha sustentado, hasta el día de hoy, las acepciones 
dominantes legitimadoras del statu quo (Acosta, 2013; Arkonda et al., 2012). Salta a la vista enseguida la enorme dificultad que implica tratar de buscar, en ese río revuelto de ideas y principios, un mínimo denominador común —más allá de su oposición discursiva ante el poder establecido y ante la colonialidad del saber que lo sustenta (Quijano, 2011) - que le dé organicidad y coherencia. De hecho, parecería que el sumak kawsay se ha convertido en una suerte de cajón de sastre capaz de albergar concepciones muy distintas - a veces casi antitéticas - en función del punto de vista en que se ubique el observador. (11)

Según Bretón, Cortez y García, en la práctica sumak kawsay se convierte en un concepto banalizado, utilizado para significar la redistribución de bienes pintada como reivindicación de los indígenas, pero a fin de cuentas un mero eslogan sin sentido. Preguntan Bretón, Cortez y García:

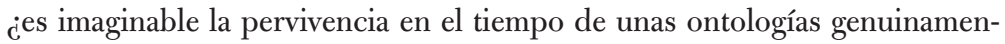
te andino-amazónicas en contextos de fuerte hibridación cultural, fruto precisamente de la subordinación y racialización secular de esos colectivos subalternos? ¿Estaremos asistiendo a una (re)esencialización de unas "culturas ancestrales" supuestamente incontaminadas como si estuvieran conservadas entre naftalinas? Si es una tradición de nuevo cuño, ¿̇se tratará de un proceso de ventriloquía (sic) política en virtud del cual algunos hablan en nombre de otros? (11-12)

Por su parte, Andreu Viola se enfoca en el sumak kawsay ecuatoriano y boliviano, "sus fuentes de inspiración intelectual, sus excesos retóricos, sus silencios y, sobre todo, su posible relevancia para el mundo rural andino contemporáneo" (58). Sugiere que

no podemos compartir la euforia de aquellos autores que han celebrado la incorporación del sumak kawsay a la nueva Constitución ecuatoriana como un "hito trascendental" para "re-fundar" el Estado y la sociedad, y capaz de abrir una nueva concepción del mundo en abierta ruptura con los esquemas eurocéntricos, coloniales y capitalistas de los 200 años precedentes (Walsh, 2009: 228). (61)

Viola contrasta la retórica de la sostenibilidad en las constituciones boliviana y ecuatoriana con las acciones extractivas concretas en el sector minero y agrario promovidas por los gobiernos de Correa y Morales. Subraya el carácter inventado del sumak kawsay por unos intelectuales indígenas y filoindianistas 
que retratan una cosmovisión idealizada contrapuesta a la visión desarrollista occidental, y enfatiza que

la notable ambigüedad e inconcreción con la que dicho buen vivir ha sido definido hasta el momento - tanto por parte de sus ideólogos como de los textos constitucionales ecuatoriano y boliviano- acarrea ciertos riesgos, siendo uno de los más evidentes la posibilidad de que este acabe convirtiéndose en un "concepto-ameba"; es decir, en una categoría de uso tan omnipresente e indefinido que llegue a quedar vacía de significado, actuando como una muletilla inofensiva, a la cual cada interlocutor pueda atribuir la connotación que le convenga, tal como parece haber sucedido durante las últimas décadas con el concepto de "desarrollo sostenible". (68)

A pesar de su difusión entre muchos actores políticos indígenas y afrodescendientes en América Latina en los últimos años, "este mimetismo no está exento de contradicciones, puesto que el valor potencial del sumak kawsay reside precisamente en su carácter local, es decir, en su arraigo en un contexto sociocultural concreto, y este, obviamente, no puede ser exportado automáticamente junto con el concepto" (68). Además, la idealización de las vivencias, prácticas y cosmovisiones de los indígenas corre el riesgo de opacar el mundo rural andino real, "minimizando sus tensiones internas y las serias amenazas que se ciernen sobre él en la actualidad" (68-69): "Alcida Rita Ramos analizó el proceso a través del cual, a partir de los años ochenta diversas ONG ecologistas o neoindigenistas del Brasil habían construido y difundido la imagen de un 'indio hiper-real' de cartón-piedra (una versión postmoderna del 'buen salvaje' rousseauniano), la pura y estilizada alteridad" (69).

Mignolo y otros decolonialistas buscan un "tercer camino" entre el capitalismo y el comunismo, pero no dan detalles más allá de una apelación vaga a una cosmogonía indígena idealizada como antídoto contra el eurocentrismo, un mundo supuestamente de "complementariedad" sin jerarquías y clases. Su postura parece ser a veces la mera inversión de la teoría nefasta de Samuel Huntington, es decir, una visión cultural binaria del mundo. Los decolonialistas establecen un tipo de lucha simbólica entre formaciones hegemonizantes rivales - el marxismo y la decolonialidad - que luego les permite evitar discursos sobre la sociedad de clases. Por ejemplo, Mignolo quiere reducir al campesinado indígena de Bolivia y de otros países andinos y mesoamericanos a una condición exclusivamente "indígena", cuando también tiene la condición de subyugado dentro de un sistema clasista y de estructuras semifeudales desde hace siglos y antes. El sistema no desaparece simplemente porque uno apele a valores precolombinos. Y Mignolo tampoco quiere 
llamar la atención a su propia posición (ni la de otros académicos decoloniales) en el sistema de clases como profesor en una universidad, con todo el capital intelectual y cultural que esto conlleva. A este respecto, resulta irónico que Ramón Grosfoguel diga: "Los marxistas y psicoanalistas todavía tienden a producir conocimiento desde el punto cero, es decir, sin cuestionar el lugar del que hablan y producen este conocimiento" ("Decolonizing" 89, traducción mía). La misma acusación podría extenderse al propio Grosfoguel y a Mignolo: la cuestión del lugar desde el que uno habla no puede ser convenientemente olvidada solo porque uno se sienta un "bárbaro" latinoamericano, mientras hace uso de todos los privilegios del primer mundo que se acumulan con un trabajo en una prestigiosa universidad (Berkeley y Duke). La rendición de cuentas epistemológicas (epistemological accountability) no cesa porque se sea un intelectual inmigrante.

En Local Histories/Global Designs, Mignolo explica la diferencia colonial latinoamericana desde el punto de vista de una experiencia sensual, vivida, y relacionada con el territorio: "las sensibilidades de las localizaciones geohistóricas tienen que ver con un sentido de territorialidad... e incluyen lengua, comida, olores, paisaje, clima y todos los signos básicos que ligan el cuerpo con uno o varios lugares" (191, traducción mía). Es difícil disputar esta afirmación. El reconocimiento de esto sería el equivalente de standpoint theory, una teoría desarrollada por feministas con su origen en Hegel (la meditación de este sobre la relación entre amos y esclavos). Pero crucialmente la teoría standpoint hace resaltar la manera como cada persona experimenta esas sensibilidades en relación con su posición socioeconómica y su capital cultural e intelectual. Infortunadamente, Mignolo borra la distancia entre los amerindios y "los blancos, los mestizos y los intelectuales inmigrantes criollos como Kush, Dussel" (Mignolo, Local 241) y él mismo, al parecer inconsciente de la objeción obvia de que hay una gran diferencia de clase, estatus y etnia entre el subalterno (indígena, afrodescendiente, etc.) y el letrado intelectual (mestizo o no) que puede participar en debates culturales euro-latinoamericanos y ganarse la vida con esa participación.

En The Idea of Latin America (2005), Mignolo instala una nueva generalización, un "universal" latinoamericano decolonial, si se quiere, al mismo tiempo que desconstruye el concepto de "América Latina". La política de la localización no puede superar la misma categoría macro-geo-cultural abstracta — "América Latina" - reconstituida con nuevo ropaje: ahora no como un área de estudios de los Estados Unidos en gran parte desacreditada, sino como el sitio de la "diferencia colonial", en el cual los interlocutores privilegiados son (una vez más) la intelectualidad letrada local. Mignolo asume una identidad inmigrante en los Estados Unidos, un país con tendencias imperialistas, como sabemos. Luego piensa 
que esto le permite reclamar una identidad oposicional, que a su vez le permite alinearse con los subalternos indígenas y afrodescendientes bajo el rótulo de "bárbaros". Pero mientras la palabra "bárbaro" sirve como término operativo, una versión redentora, similar a la apropiación del nombre de "Calibán" por José Martí y Roberto Fernández Retamar para los desgraciados de la tierra, como los llama Frantz Fanon (Les damnés de la terre), es ilegítimo que un intelectual se apropie del mismo rango o posición de clase.

Scott Michaelsen y Scott Shershow identifican tres contrastes fundamentales u oposiciones conceptuales "cuya interacción es crucial para el trabajo de Mignolo: 1) las prácticas de significación amerindias versus las europeas; 2) la complementariedad amerindia versus el 'binarismo' o 'dualismo' europeo; y 3) la democracia amerindia versus la nación-Estado europea" (40, traducción mía). Para Michaelsen y Shershow, todos los análisis específicos de Mignolo se basan en una nostalgia por una Arcadia epistemológica y política que a fin de cuentas socava su proyecto. Estas contradicciones llevan a Mignolo a desechar o descartar la teoría desconstructiva de Derrida, por ejemplo, por estar contaminada del pensamiento crítico europeo derivado de la Ilustración. La crítica del logocentrismo de Derrida expone la falacia de una filosofía que postula que las formas de pensamiento tienen un punto de referencia externo que les da cierto grado de autoridad. La desconstrucción de este mito tiende a socavar todos los autoritarismos que se basen místicamente en la palabra definitiva, original, fuera de la historia. Para Derrida, el logocentrismo es también el etnocentrismo, y así la desconstrucción derrideana bien podría fundamentar la teoría de los decolonialistas y servir como freno contra su propia ceguera y sus propias fantasías. Pero Mignolo está tan ansioso de distanciarse de todo lo europeo, lo occidental (que ve sin matices como algo fundamentalmente imperialista), que propone que la "metafísica occidental" no tiene nada que ver con los sujetos coloniales ni tiene nada que decir sobre ellos. Sostiene que Derrida es ciego a "la otredad epistemológica y ontológica radical de tales sujetos" y "extender la desconstrucción más allá de la metafísica occidental [...] es una maniobra similar a los diseños globales colonizadores" (Mignolo Local 326 ).

Retomando el hilo de nuevo con Derrida en el posterior Writing without Words (Escribir sin palabras), Mignolo dice que "los sistemas antiguos amerindios de escribir son totalmente ajenos a la idea de la escritura como una representación del habla" (302), como lo propone Derrida. Pero según Michaelsen y Shershow, los "primeros textos [de Derrida] critican rigurosamente todas las explicaciones teleológicas y evolucionistas en las ciencias humanas" (5, traducción mía). Así, Mignolo malinterpreta a Derrida cuando dice que según este el alfabeto superó 
formas alternativas o prealfabéticas de escribir. Lo que Derrida dice es que las formas no-alfabéticas son, de hecho, formas de escribir en el sentido de significar. Son inteligibles y comunicables. Además, según Pheng Cheah, "las lenguas indígenas no son inherentemente igualitarias o liberadoras solo por ser no europeas. Las lenguas no europeas pueden tener registros jerárquicos, conservadores o reaccionarios" (11, traducción mía). Parece obvio entonces que lo que motiva a Mignolo es el deseo de presentarse como un pensador de alguna manera original que ha creado una contrateoría al pensamiento eurocéntrico. Pero uno no tiene derecho a hacer eso atribuyéndole a Derrida algo que no dijo. Los críticos decoloniales hablan con una autoridad injustificada. Su estilo es declamatorio y, a pesar de toda la tinta derramada sobre la "pluriversalidad", practican una especie de crítica monológica y compartimentada sobre lo que definen como la Modernidad, el Occidentalismo y la Razón Ilustrada. El precio pagado por dicho rechazo del pensamiento crítico europeo es dejar sus propios argumentos sin controles de autorreflexión y evaluación por parte de sus pares intelectuales críticos, aunque generen sus discursos y opiniones desde dentro de otra serie de universalismos (y desde dentro de las universidades, una invención europea).

Algo del sabor de esta autocuarentena de la reflexión crítica decolonial se puede extraer de la reciente introducción escrita por Mignolo, melodramáticamente titulada "The Point of Nonreturn: The Reemergence of the Disavowed" (El punto de no retorno: el resurgimiento de lo desautorizado), para la renacida revista Comparative Studies of South Asia, Africa and the Middle East (CSSAAME): [...] En este sentido, CSSAAME tiene una enorme contribución que hacer en la construcción de futuros globales. La pregunta sigue siendo: ¿Cómo podría un diario basado en los Estados Unidos descentrarse? [...] ¿ $]_{\mathrm{C}}$ Puede CSSAAME trascender y transgredir las restricciones académicas y publicar ensayos y declaraciones que, en lugar de seguir las normas académicas, se hagan con la fuerza y las demandas de la esfera pública? Una de las características de la época actual no es solo que los estudios de área están fuera de lugar, sino también que la erudición normativa como una forma segura y objetiva del conocimiento es sospechosa. (269, traducción mía)

Luego, Mignolo amonesta con dedo acusador como un sabio padre: "El título mismo de esta revista fue testigo de la complicidad entre la comparación y la distribución del trabajo científico que, estoy seguro, CSSAAME está comprometida a superar" (270).

A pesar de su reciente ataque contra Mignolo y Quijano (sintomático de las contradicciones del ideario decolonial) (Martínez, "Entrevista"), Ramón 
Grosfoguel peca de las mismas exageraciones y binarios. Pretende demostrar que el racismo y el patriarcado son exclusivos del mundo europeo o que, si ocurren en otras partes, es a causa de la modernidad europea. Y no obstante sus llamados a la "diversalidad epistémica", al igual que otros decolonialistas, no ofrece detalles: "como política occidental sistemática y coherente nunca vemos respeto o reconocimiento por las formas de las democracias indígenas, islámicas o africanas" ("Decolonizing" 97). Esto puede ser cierto en gran medida, y no sería la primera vez que una cosmovisión eurocéntrica culturalmente sorda les ha faltado al respeto a otros pueblos, pero ${ }_{\mathrm{C}}$ cuáles son esas otras formas de la democracia, si es que desean utilizar la palabra "democracia" (un concepto occidental)? Grosfoguel pide "una comunicación verdaderamente igualitaria y horizontal y el diálogo entre los pueblos del mundo que va más allá de las lógicas y prácticas de dominación y explotación características del sistemamundo eurocentrado" ("Decolonizing" 98). De acuerdo. Pero cipor qué no ir más allá todavía y abogar por el fin de todas las dominaciones y explotaciones, incluso las que preceden a la expansión europea y ocurren al mismo tiempo y después en áreas no europeas? El problema es que los decolonialistas han universalizado la fuente de toda dominación y mal: Europa. Pero si aceptamos que el colonialismo es la extensión de control, típicamente por la violencia, sobre otro territorio y otro grupo humano y el establecimiento de relaciones de tributo, entonces el colonialismo ha sido universal por milenios. La historia general de la humanidad, atravesada a todo lo largo por diferencias étnicas y culturales, es una guerra tribal desde mucho antes de la colonización europea. Esto no exculpa a Europa y su historia colonial (ni la eximen de las deudas aún no pagadas), pero uno no se puede quedar convenientemente mudo frente a esos aspectos de las historias de los otros para proteger sus propios argumentos. Grosfoguel dice:

Habrá tantas soluciones como proyectos ético-epistémico-políticos en el mundo [...] "La universalidad positiva" implicaría reproducir desde la izquierda una vez más el concepto occidental-céntrico, el problemático concepto de lo "universal" descrito en este artículo. El "pluri" en contraposición a la "uni" no es apoyar todo lo dicho por un sujeto subalterno de abajo, pero sí una llamada a producir conocimiento decolonial crítico que sea riguroso y completo, con alcance mundial y no provincial. ("Decolonizing" 101-102)

Pero ¿qué es un pensamiento de "alcance mundial" y "no provincial" si no un universalismo? No se puede criticar los impulsos y demandas de universalización en el pensamiento occidental y luego reclamar un conocimiento decolonial 
autárquico no sujeto a las mismas normas de la crítica, a pesar de la proliferación de neologismos exuberantes como "diversalidad", "pluritópico", "diferencia colonial", etc. Parece que las normas filosóficas se cambian a gusto en cada paso, pero no se puede estar en misa y repicar a la vez.

De manera similar, en "Latin American Philosophy as Critical Ontology of the Present: Themes and Motifs for a "Critique of Latin American Reason"" (2003), Santiago Castro-Gómez también quiere desconstruir las nociones de "América Latina" y "modernidad":

¿Cómo ha sido construido un orden específico de las palabras y las cosas a partir del cual hemos generado conocimiento sobre "qué es lo propio" y lo "extranjero" en América Latina? Esto se refiere, sin duda, a una cuestión que provoca un cierto malestar en algunos sectores de la comunidad intelectual latinoamericana. Nos hemos acostumbrado a pensar en América Latina como un lugar de la utopía y el realismo mágico, como el sitio de un proyecto que es autóctono y que presenta un proyecto alternativo a la modernidad occidental, o como un espacio de carencias y ocultaciones que nos han impedido llegar a la "verdadera modernidad" lograda por Europa y Estados Unidos. Pero la pregunta de Salazar Ramos no está dirigida al "ser" de América Latina, o a las "bases normativas" de la modernidad, sino al orden epistemológico-social que ha hecho posible la construcción de objetos de conocimiento como "América Latina", "Occidente", "Europa" y "modernidad". La pregunta establece, entonces, una línea de fuga con respecto a la episteme cuyo problema ha sido formulado por algunos: ambas partes insistieron en cuestionar el acceso de América Latina a la modernidad, sin reconocer que ambas categorías no denotan absolutamente nada fuera del orden simbólico en el que fueron construidas. (72-73, traducción mía)

Verdad. De hecho el argumento es muy derrideano: cualquier construcción de lenguaje representativo o proceso de significación solo puede funcionar al nombrar las cosas y adjuntar a los nombres significados dentro de un contexto dado comunicable. Pero la comprensión de esto tiene que extenderse a todas las construcciones lingüísticas conceptuales, incluidas las de la teoría decolonial y sus neologismos, denotados dentro "del orden simbólico en el que fueron construidos". No se puede tener dos series de reglas: elaborar un discurso teórico que utiliza la crítica occidental para desconstruir las categorías de la filosofía occidental y luego convenientemente detenerse allí, como si los conocimientos indígenas o afrodescendientes y sus jerarquías sociales y la manera en que sus historias se construyen fueran naturalmente no problemáticos e intocables 
simplemente porque han sido reprimidos y explotados por los europeos (algo que no está en disputa). Filosofar así crea visiones del mundo mutuamente incomprensibles y la imposibilidad de la comunicación que en últimas obligaría a los decolonialistas mismos a estar en silencio. En otras palabras, no se pueden proyectar sobre los pueblos indígenas y afrodescendientes los propios deseos como intelectual local radicalizado, y poner en cuarentena las propias construcciones de conocimiento sin entrar en contradicción performativa.

Castro-Gómez continúa: "Es inútil llorar y colocar flores en la tumba de los proyectos históricos, como la 'autarquía' y la 'modernización', que han sido suplantados por los procesos de transnacionalización económica y cultural" (74). Sí; y Castro-Gómez debería hacer una pausa antes de alinearse con los teóricos decoloniales que estereotipan a los pueblos indígenas como necesariamente antimodernos o anti-Europa, como intrínsecamente diferentes a los "los occidentales", solidarios e igualitarios en vez de competitivos; pacíficos, generosos y holísticos por naturaleza. Hay un maniqueísmo en la manera como los decolonialistas retratan y yuxtaponen una subjetividad no-europea transparente, estable y no-problemática (indígenas, afrodescendientes, mujeres, homosexuales, etc.) al hombre blanco heterosexual de Occidente. Pintan tal subjetividad del otro como igualmente estática, como si existiera algún tipo de subjetividad subalterna autóctona, estable y generalizada lista para ser redimida. Pero todas las subjetividades son fenómenos en estado de cambio y adaptación constantes dentro del contexto en el cual operan, por pequeño que sea el cambio en las subjetividades poco occidentalizadas y no tan sujetas a las veloces mutaciones de la modernidad. Semejantes binarios opuestos de los decolonialistas son caricaturas y violentan simbólicamente la subjetividad compleja de todos, no importa de qué lado del binario rígido se encuentren. También queda fuera de la crítica decolonial el disciplinamiento de esos otros no-europeos dentro de sus propias sociedades - las sociedades indígenas eran adeptas a disciplinar los cuerpos y mentes (por lo menos en las civilizaciones sedentarias grandes) para mantener la jerarquía vertical: estructuras socioeconómicas piramidales con gobernantes, sacerdotes, guerreros, labradores, etc.- .

Si el proyecto de la modernidad requiere la formación de las estructuras que los actores sociales reproducen, la globalización abre los barrotes de la "jaula de hierro" y proyecta la imagen de las estructuras que los propios actores transforman. La dialéctica moderna entre el sujeto y la estructura pierde empuje y comienza a "debilitarse" - como Vattimo diría - de tal manera que las estructuras se convierten en el objeto de los procesos de acción y el cambio social. (Castro-Gómez 77) 
Es difícil entender lo que Castro-Gómez dice aquí; es confuso. La modernidad reclamaría eso también, no únicamente la imagen de un solo lado de la modernidad proyectada por sus críticos. Además, no está claro que la "globalización" (una palabra eurocéntrica igualmente nebulosa) sea otra cosa que una etapa avanzada de la modernidad occidental sin algunos de los controles que normalmente tiene bajo el mando de las naciones-Estado (el discurso de la modernización socioeconómica occidental, por ejemplo, sigue más o menos intacto).

Entonces, la manera en que los teóricos de la decolonialidad a veces se apropian del mundo indígena o afrodescendiente para beneficio de su discurso parece pecar del mismo esencialismo y binarismo. Una alternativa a este tipo de teoría, o una apropiación más sutil, se encuentra en el trabajo del indígena intelectual australiano Martin Nakata. A través de Nakata queda más claro lo que realmente está en juego cuando invocamos el mundo no-europeo en el discurso decolonial. Nakata promueve lo que llama "la interfaz cultural" y su preocupación principal es la educación indígena y la educación sobre lo indígena. Dice: "La interfaz cultural está constituida por puntos de trayectorias que se intersectan [...] Es un espacio de muchas intersecciones cambiantes y complejas entre diferentes personas con diferentes historias, experiencias, lenguas, agendas, aspiraciones y respuestas" (Disciplining 199, traducción mía).

El discurso global sobre el conocimiento indígena atraviesa una serie de intereses como el desarrollo sostenible, la biodiversidad y la conservación, los intereses comerciales y corporativos. Circula en los ámbitos internacional, nacional, estatal, regional y local en sectores gubernamentales, no gubernamentales y de comunidades indígenas, así como entre una serie de agendas intelectuales, públicas, privadas e indígenas. De modo que "debemos ser muy cuidadosos en esto. No todo es blanco o negro, y las cosas no se arreglan con solo añadir a la mezcla el componente indígena. Este es un espacio muy complejo y disputado" (Disciplining 10). En un artículo más reciente, de 2012, "Metas y pedagogías decoloniales para los estudios indígenas", Nakata arguye que "los estudios indígenas se han fijado en una descolonización simplista del conocimiento y las prácticas occidentales. Queremos convertir en una prioridad el desarrollo de disposiciones de aprendizaje en los estudiantes; esas disposiciones deben alentar la apertura hacia más investigación y maneras productivas de pensar en terrenos de conocimiento complejos e impugnados" ("Decolonial" 120, traducción mía).

En las universidades australianas, la razón decolonial ha alcanzado cierta legitimidad como marco docente y como una teoría que se compagina con la política de la autodeterminación de los pueblos indígenas y aun con el marco de justicia social en la política del Gobierno australiano. Nakata reconoce las 
sinergias con los acercamientos decoloniales latinoamericanos. Sin embargo, "la crítica de Occidente no es suficiente. Un imperativo de la decolonialidad, y una tarea central para los indígenas, es 'el hacer conocimiento decolonial indígena' que reafirme e incluya conceptos y significaciones del conocimiento y los sistemas de pensamiento indígenas. Esto hace una interfaz compleja" ("Decolonial" 124). Y es más: si los indígenas quieren de veras exponer y criticar las fuerzas de dominación y los modos a través de los cuales se establecen, entonces, se pregunta Nakata, "¿no debemos estar más abiertos a la cuestión de cuáles opresiones indígenas no podemos interrogar cuando el hacer conocimiento decolonial indígena asume una ceguera epistemológica hacia sus propias prácticas?" (130). Así, Nakata aprueba la postura de Deloria, quien comenta la situación norteamericana: "los indígenas tienen que examinar algunos de los mismos fenómenos como pensadores occidentales y deben demostrar que sus perspectivas y conclusiones tienen sentido" (cit. en Nakata, "Decolonial" 128). Nakata dice que esto puede parecer injusto dado que los indígenas están luchando todo el tiempo contra la opresión. No obstante,

allí donde el saber es autorizado por vía de afirmaciones de epistemologías y "tradiciones" indígenas para invalidar y oponerse a las construcciones coloniales/occidentales, los enredos menos examinados donde lo occidental y lo indígena convergen y se constituyen mutuamente merecen mucha más investigación y análisis, especialmente en términos de cómo entendemos la vida cotidiana indígena. (129)

Afirma que "la defensa de lo indígena no necesariamente depende de la autentificación o la separación de lo indígena apelando a nociones de 'soberanía intelectual' y resistencia a lo occidental" (131). Entonces, cuando uno escucha esto de un intelectual indígena que ha luchado por años contra la opresión, la exclusión y/o asimilación de los indígenas australianos, hay que prestar atención, ¿no? Nakata concluye:

En la compleja interfaz indígena-occidental, las "formas de escepticismo y actitudes epistémicas" son necesarias para considerar las delimitaciones y disposiciones tanto de las teorizaciones indígenas como de las occidentales sobre las realidades sociales contemporáneas indígenas en este espacio y así las posibilidades para el futuro [...] Los estudiantes necesitan más que herramientas analíticas y lingüisticas para una crítica simplista y un marco decolonial que precipitadamente los colocan en el binario occidente-indígena. (131, énfasis añadido) 
La complejidad de la interfaz cultural entre los distintos grupos y el histórico acondicionamiento global impulsado por la "modernidad" (comoquiera que se la defina) y sus efectos colonizadores significa que no podemos quitárnosla de encima conveniente y voluntariamente como si fuera simple ropa sucia, no importa cuán perjudiciales hayan sido sus efectos. Para Pheng Cheah, quien entrevistó a Mignolo en 2006, "el estado actual de las relaciones de poder es un efecto de procesos múltiples que son dinámicos, heterogéneos e inestables. Procesos que no pueden ser reducidos a una sola lógica de colonialidad, aunque esta última puede surgir como su efecto" (11). En consecuencia, debemos rechazar tanto los particularismos militantes como las proposiciones universales falsas, algo con lo cual Grosfoguel parece estar de acuerdo. Sin embargo, aunque se encuentran divergencias en las propuestas de los decolonialistas, da la impresión de que la mayoría adhiere a una versión maniquea de la modernidad europea y, en el caso de Mignolo, a una visión esencialista de los indígenas. Lo que consideran crítica (critique) es solo el estar en comunión con grupos subalternizados, el proponer sus vivencias y sus cosmogonías como mejores simplemente por ser subalternos.

En tiempos recientes la teoría decolonial ha asumido el estatus de un culto, casi una secta religiosa, con sus devotos y sumos sacerdotes carismáticos que hablan con una autoridad auto-otorgada basada en un antieuropeísmo sin matices. Pero muchos de sus argumentos no resisten el más mínimo examen. Los intelectuales decoloniales quieren utilizar aspectos de la teoría crítica que tiene raíces en la epistemología de la Ilustración (que tiene una historia de más de 300 años en Europa y por supuesto América Latina), pero al mismo tiempo ponerla entre corchetes y salir de la modernidad a través de su contacto con el mundo indígena o afrodescendiente, que es equivalente a ascender al cielo por una escalera y luego subirla, un malabar difícil, ¿no? Una crítica convincente de la modernidad, su bagaje conceptual y sus genealogías pierde legitimidad si el punto de vista epistemológico del discurso propio, con su locus de enunciación, su arquitectura terminológica y sus maniobras discursivas, no recibe una concomitante crítica autorreflexiva y severa. Las cuestiones epistemológicas no desaparecen mágicamente porque el intelectual latinoamericano proponga algún místico "espacio fronterizo" epistemológicamente liberado mientras está instalado en una universidad occidental dentro o fuera de América Latina. Esto es una estrategia de legitimización en busca del reconocimiento. No se puede hacer las dos cosas: desconstruir solo lo europeo y luego re-inventarse a sí mismo como alguien que reside afuera, en un espacio conceptual autárquico, y no permitir la desconstrucción de su propia ficción. 


\section{Obras citadas}

Bretón, V., D. Cortez y F. García. "En busca del sumak kawsay". Íconos. Revista de Ciencias Sociales 48 (enero de 2014): 9-24.

Browitt, J. y N. Castrillón, trads. Disciplinar a los salvajes, violentar las disciplinas. Martin Nakata. Canberra: Aboriginal Studies Press; Quito: Abya-Yala, 2014, en prensa.

Castro-Gómez, Santiago. "Latin American Philosophy as Critical Ontology of the Present: Themes and Motifs for a 'Critique of Latin American Reason"'. En Latin American Philosophy: Currents, Issues, Debates. Ed. Eduardo Mendieta. Bloomington: Indiana University Press; 2003, 68-79.

Castro-Gómez, Santiago y Ramón Grosfoguel, eds. El giro decolonial. Reflexiones para una diversidad epistémica más allá del capitalismo global. Bogotá: Siglo del Hombre Editores, 2007.

Chanady, A., ed. Latin American Identity and Constructions of Difference. Minneapolis: University of Minnesota Press, 1994.

Cheah, Pheng. "The Limits of Thinking in Decolonial Strategies". Townsend Newsletter noviembre-diciembre de 2006: 9-11.

Deloria, V.Jr. "Philosophy and the Tribal Peoples". En American Indian Thought: Philosophical Essays. Ed. A. Waters. Malden, M.A.: Blackwell Publishing, 2004. 3-11.

Domingues, José Maurício. "Global Modernization, 'Coloniality' and a Critical Sociology for Contemporary Latin America". Theory Culture Society 26.1 (2009): 112-133.

Grosfoguel, Ramón y José David Saldívar, eds. Unsettling Postcoloniality, Coloniality, Transmodernity and Borderthinking. Durham, NC: Duke University Press, 2004.

Grosfoguel, Ramón. "Decolonizing Western Uni-versalisms: Decolonial Pluriversalism from Aimé Césaire to the Zapatistas". Transmodernity: Fournal of Peripheral Cultural Production of the Luso-Hispanic World 1.3 (2013): 88-104.

—. "Transmodernity, Border Thinking, and Global Coloniality: Decolonizing Political Economy and Postcolonial Studies". Eurozine 28 de julio de 2008. Web. 2013.

Maldonado-Torres, Nelson. "Decoloniality at Large: Towards a Trans-Americas and Global Transmodern Paradigm (Introduction to Second Special Issue of 'Thinking Through the Decolonial Turn')". Transmodernity: Fournal of Peripheral Cultural Production of the Luso-Hispanic World 1.3 (2012): 1-10.

Martínez Andrade, Luis. "Entrevista a Ramón Grosfoguel". Analéctica 1 de noviembre de 2013. Web. 13 de diciembre de 2013.

- . "Ramón Grosfoguel: 'Hay que tomarse en serio el pensamiento crítico de los colonizados en toda su complejidad"' (entrevista). Metapolítica 17.83 (2013): 38-47. 
Michaelsen, Scott y Scott Cutler Shershow. "Rethinking Border Thinking". Latin America in Theory. Número especial de South Atlantic Quarterly 106.1 (2007): 39-60.

Mignolo, Walter. "Cosmopolitanism and the De-Colonial Option".

Studies in the Philosophy of Education 29 (2010): 111-127.

-. "Delinking: The Rhetoric of Modernity, the Logic of Coloniality and the Grammar of De-Coloniality". Cultural Studies 21.2-3 (2007): 449-514.

-. "Epistemic Disobedience, Independent Thought and Decolonial Freedom". Theory, Culture \& Society 26.7-8 (2009): 159-181.

-. The Idea of Latin America. Oxford: Blackwell, 2005.

-. Local Histories/Global Designs: Coloniality, Subaltern Knowledges and Border Thinking. Princeton: Princeton University Press, 2000.

-. "The Point of Nonreturn: The Reemergence of the Disavowed". Comparative Studies of South Asia, Africa and the Middle East (CSSAAME) 33.3 (2013): 268-271.

Nakata, Martin. "Decolonial Goals and Pedagogies for Indigenous Studies".

Decolonization: Indigeneity, Education \& Society 1.1 (2012): 120-140.

-.Disciplining the Savages: Savaging the Disciplines.

Canberra: Aboriginal Studies Press, 2007.

-. "La interfaz cultural". Trad. Débora McLauchlan. Revista

Peruana de Investigación Educativa 1.2 (2010): 7-26.

Reasens, A. V. "Discursos "pachamamistas" versus políticas desarrollistas: el debate sobre el sumak kawsay en los Andes". Íconos. Revista de Ciencias Sociales 48 (2014): 55-72.

Viola, Recasens Andreu. "Discursos 'pachamamistas' versus políticas desarrollistas: el debate sobre el sumak kawsay en los Andes". Íconos. Revista de Ciencias Sociales 48 (2014): 55-72.

Walsh, Catherine. "Interculturalidad, decolonialidad y el buen vivir". Interculturalidad, Estado, sociedad. Luchas (de)coloniales de nuestra época. Ed. Catherine Walsh.

Quito: Abya-Yala; Universidad Andina Simón Bolívar, 2009; 213-235. 